\section{The symptomatic Pulfrich \\ phenomenon can be successfully managed with a coloured lens in front of the good eye-a long-term follow-up study}

G Heron ${ }^{1}$, KJ Thompson ${ }^{2}$ and GN Dutton ${ }^{1,2}$

\begin{abstract}
Purpose Many visual symptoms originating from a spontaneous Pulfrich phenomenon can be alleviated by the provision of a tinted lens worn in front of the least affected eye. Here, we report on the long-term outcome of four patients who have been provided with a uniocular tinted spectacle or contact lens. Methods The case notes of four cases were reviewed. The review period was at least 5 years in all four cases.

Results In all cases, visual symptoms and perceptual difficulties occurring from the spontaneous Pulfrich effect were completely eliminated over a period of many years. In one case, the relief has been sustained over a 20-year period.

Conclusions A uniocular tinted lens, or contact lens, provides immediate and lasting relief from the visual difficulties caused by the visual delay underlying the spontaneous Pulfrich effect.
\end{abstract}

Eye (2007) 21, 1469-1472; doi:10.1038/sj.eye.6702459; published online 9 June 2006

Keywords: Pulfrich phenomenon; treatment; management

\section{Introduction}

Patients with optic neuropathy, for example due to multiple sclerosis, may describe bumping into things and a sensation that oncoming targets or vehicles appear to veer towards them at the last moment. Delayed conduction from one eye to the brain leads to an altered appreciation of the pathway of oncoming targets approaching the affected side. A pendulum, swung to and fro in front of the patient's face, is seen to transcribe an oval when viewed binocularly, but to follow a straight line when viewed monocularly. This condition comprises the Pulfrich phenomenon. Figure 1a illustrates how the apparent oval path is brought about, and Figure 1b shows how an oncoming target approaching the right affected eye appears to move in an arc towards that eye. The Pulfrich phenomenon facilitates diagnosis of optic neuropathy and is a cause of potentially hazardous symptoms. ${ }^{1}$ Symptomatic relief can be afforded by spectacles with one tinted lens (or a tinted contact lens) in front of the unaffected eye. The tint prolongs retinal processing time to match the delay on the other side. ${ }^{2}$ We report the long-term follow-up of four patients who have been managed in this way.

\section{Case reports}

\section{Patient 1}

A 30-year-old nightclub manageress had experienced two episodes of optic neuritis owing to multiple sclerosis. Her visual difficulties included difficulty walking down stairs owing to misjudging depth, pouring tea into the saucer instead of the cup, bumping her car into kerbs, and misjudging the positions of other moving cars.
${ }^{1}$ Department of Vision Sciences, Glasgow Caledonian University, Glasgow, UK

${ }^{2}$ Tennent Institute of Ophthalmology, Gartnavel General Hospital, Glasgow, UK

Correspondence:

GN Dutton,

Tennent Institute of Ophthalmology, Gartnavel General Hospital, 1051 Great Western Road, Glasgow G12 8QQ,

Scotland, UK

Tel: + 44141331 3389;

Fax: + 441413313387

E-mail: dutton@

ledcresc-adsl.demon.co.uk

Received: 21 March 2006 Accepted in revised form: 23 April 2006 Published online: 9 June 2006

The authors have no competing interests

Contributors: KT reviewed the clinical data. GH and GD diagnosed and managed the patients and drafted the manuscript, which was edited by all parties 

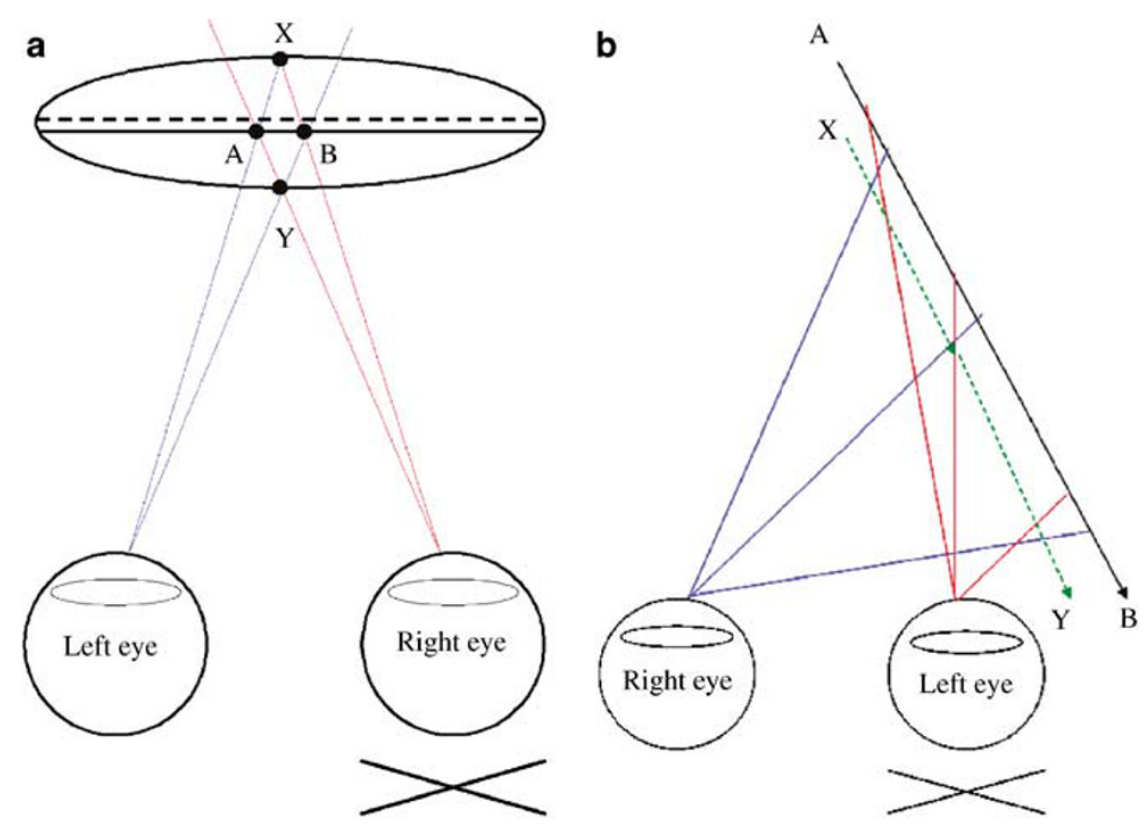

Figure 1 (a and b) Diagrams illustrating how a moving target is perceived. The left eye is normal, but the right has slow conduction to the brain, denoted by the cross behind the right eye. The left eye 'sees' the target before the right eye. The intersections of the lines projected from the two eyes show the position of the pathway of targets as seen by the patient. (a) A pendulum swung across the patient's line of gaze is seen to follow an oval path. As the pendulum moves from A to B, it is seen at $Y$, and as it moves from B to A, it is seen at X. Thus, the apparent oval path of the pendulum is seen as being anticlockwise. The actual path of the pendulum is shown by the dotted line. When the left eye is affected, matters are reversed and the oval path of the pendulum is seen as being clockwise. (b) An oncoming target travelling from A to B appears to veer towards the observer's affected eye. The apparent path of the target is indicated by the green dotted lines.

A simple pendulum swinging at right angles to her line of gaze appeared to move in a clockwise ellipse. This suggested conduction delay along the left optic nerve, confirmed by visual-evoked potentials. There was no detectable afferent pupil deficit. The apparent elliptical pathway of the pendulum was eliminated using a slight tint ( $85 \%$ transmission) in front of the right eye, incorporated into a spectacle correction. She has worn these glasses successfully for 5 years. Despite episodes of relapse of her multiple sclerosis, restoration of normal vision has been maintained. This has enabled her to continue driving.

\section{Patient 2}

A 40-year-old police driver was assaulted and sustained a right malar fracture. He subsequently suffered headaches and double vision. He was aware of reduced light brightness appreciation in the right eye, and diplopia on right-gaze and up-gaze, which has persisted. His other visual symptoms comprise an inability to play table tennis, badminton, and football (because he missed the ball or shuttlecock), and difficulties driving. He had twice damaged the left side of his car, by veering too far to the left to avoid oncoming cars on his right. On examination, an apparent anticlockwise pathway of the pendulum indicated conduction delay of the right optic nerve due to presumed mild traumatic optic neuropathy. A filter with $75 \%$ transmission in front of the left eye eliminated the illusion, and was incorporated into a spectacle correction. This has eliminated all his visual perception errors. He had been stopped from police driving, but wearing his spectacles he passed a reassessment and resumed his career. He has worn his glasses for driving for 6 years.

\section{Patient 3}

A 24-year-old student was involved in a road traffic accident. Witnesses noticed that he did not take sufficient action to avoid the path of an oncoming lorry on his right side. He was known to have multiple sclerosis and had had a prior episode of left retrobulbar neuritis. He complained of difficulty in judging distances on the lefthand side. Objects on the left, including pedestrians, seemed to be too close. Passengers in the car observed that he drove near the centre of the road. On examination, the errors in perceived depth were evident when viewing a simple pendulum. A clockwise ellipse was seen, and was eliminated using a $76 \%$ transmission 
filter in front of the right eye. The management was complicated by relapse of his multiple sclerosis. Soon after he collected his glasses, further episodes occurred and darker tints were needed to eliminate his visual symptoms. During remission, wearing the original spectacles enabled him to drive during the ensuing 2 years, but following his latest relapse he has been unable to do so.

\section{Patient 4}

A woman presented 20 years ago, aged 48 years, with a number of visual difficulties resulting from a presumed vascular accident. This had caused a superior altitudinal right visual field defect and a positive Pulfrich effect. ${ }^{2}$ She had stopped driving. When in a car, traffic on her right appeared to swerve towards her. She had problems with her embroidery and her stitching was crooked. She misjudged the height of doorways and made errors putting crockery correctly on shelves. A tint incorporated into her left spectacle lens eliminated her symptoms and she was able to drive again. Over the ensuing 20 years, the type and density of the filter has been altered on account of cataract. Recently, no filter has been required because right cataract surgery has improved the brightness of the retinal image, but persistent left lens nuclear sclerosis has produced an intrinsic filter effect without significantly diminishing visual acuity and has, for the present, eliminated her Pulfrich effect.

All four patients have maintained visual acuities of $6 / 6$ in the normal eye and $6 / 9$ or $6 / 12$ in the affected eye during the period of study.

\section{Discussion}

\section{The Pulfrich phenomenon}

Carl Pulfrich presented a theoretical paper in 1922 showing that delay in visual processing in one eye should cause an illusion of targets moving in variance to reality. ${ }^{1}$ A darkened filter placed in front of one eye or dilatation of one pupil causes the effect, because decreased luminance increases retinal processing time. Grimshaw $^{3}$ described a patient with optic neuritis whose tennis game had deteriorated and improvement was sought using a filter in front of the unaffected eye. Conditions that cause the Pulfrich phenomenon include injury, ischaemia, or glaucomatous damage ${ }^{4}$ to the optic nerve and resolved optic neuritis. A unilateral dilated pupil, ${ }^{5}$ asymmetric cataract, and unilateral lens capsule thickening after cataract surgery may cause the problem owing to interocular differences in retinal luminance. ${ }^{6,7}$ (Readers can experience the phenomenon by viewing a pendulum moving as shown in Figure 1, with a sunglass lens in front of one eye.)

\section{Presenting symptoms}

Table 1 summarises the symptoms caused by the Pulfrich phenomenon. The problems can be difficult to describe, but a consistent history of bumping into things on one side in a patient with good vision in each eye should lead to suspicion of the diagnosis.

\section{Making the diagnosis}

The key to making the diagnosis is to be sensitive to the description of any of the symptoms described in Table 1. The examination strategy, which elicits the diagnosis most effectively, is to swing a pendulum in the directions shown in Figure 1a and b, with one eye covered and then uncovered while the pendulum is swinging. Affected individuals immediately notice a change in the apparent pathways of the pendulum in the directions shown. When the pendulum is swung diagonally towards the affected eye, immediate avoiding action is taken because of the perception that the pendulum is going to hit the face.

\section{Differential diagnosis and further investigation}

Misreaching and incoordination can be due to a wide range of causes of damage to any of the multiple brain structures responsible. Patients with a spontaneous Pulfrich effect, however, have relative delay in one optic nerve measured by visual-evoked potentials, ${ }^{8,9}$ and the perception of a pendulum to move in the directions

Table 1 Typical difficulties experienced by patients symptomatic from the Pulfrich phenomenon

Pouring liquid into vessels. The vessel is often missed.

Locating keys into locks. The key-hole is initially missed.

Judging the height and width of doors. Subjects may duck the lintel or dodge the door frame when going through doorways.

Judging height when placing objects on a surface like a table top can be difficult.

Putting objects in cupboards and drawers is difficult.

Difficulty judging the position of traffic, kerbs, street lights, and road markings relative to themselves when driving or being driven.

Navigating along pavements, corridors, shops, and precincts, and avoiding pedestrians and obstacles is difficult.

Inaccuracy steering a bicycle can be problematic.

Ascending or descending stairs can be difficult.

Positional errors in embroidery cause irregular stitching.

Increased errors occur in games like tennis or badminton with increased scores in golf. 
indicated is unique to this diagnosis. Investigation for the causative conditions described above may be required.

\section{Management}

The affected patient is asked to observe a pendulum swinging to and fro at right angles to the direction of gaze. A series of tinted filters is placed in front of the unaffected eye and the one that eliminates the illusion is chosen. The choice is confirmed by checking that no symptoms are experienced when swinging the pendulum in the directions shown in Figure 1b. A spectacle lens or contact lens with the chosen light transmission is provided for the unaffected eye. The symptomatic relief afforded by the filter is immediate and, for some, dramatic. If the underlying cause is stable, the density of filter required remains unchanged. To our knowledge, this is the first report of long-term benefit from this approach.

\section{References}

1 Diaper CJ. Pulfrich revisited. Surv Ophthalmol 1997; 41: 493-499.

2 Heron G, Dutton GN. The Pulfrich phenomenon and its alleviation with a neutral density filter. Br J Ophthalmol 1989; 73: 1004-1008.

3 Grimshaw H. A note on Pulfrich's phenomenon with a suggestion on the possible clinical importance. $\mathrm{Br} \mathrm{J}$ Ophthalmol 1925; 9: 63-65.

4 Tong DT, Borsting E, Ridder III WH. Management of the Pulfrich phenomenon secondary to pigmentary glaucoma. Optometry 2001; 72: 86-93.

5 Heron G, McQuaid M, Morrice E. The Pulfrich effect in optometric practice. Ophthalmic Physiol Opt 1995; 15: 425-429.

6 Diaper CJ, Heron G, MacMillan ES. Correction of the Pulfrich phenomenon by surgery and laser. J Cataract Refract Surg 2002; 28: 369-372.

7 Scotcher SM, Laidlaw DA, Canning CR, Weal MJ, Harrad RA. Pulfrich's phenomenon in unilateral cataract. $\mathrm{Br} J$ Ophthalmol 1997; 81: 1050-1055.

8 Rushton D. Use of the Pulfrich pendulum for detecting abnormal delay in the visual pathway in multiple sclerosis. Brain 1975; 98: 283-296.

9 Heron G, McCulloch L, Dutton GN. Visual latency in the spontaneous Pulfrich effect. Graefes Arch Clin Exp Ophthalmol 2002; 240: 644-649. 